\title{
Relaciones entre internas y guardias en el penal de mujeres de Lima: análisis de las desigualdades de trato
}

\section{Chloé Constant}

\section{(2) OpenEdition}

\section{Journals}

Edición electrónica

URL: http://journals.openedition.org/bifea/1550

DOI: 10.4000/bifea. 1550

ISSN: 2076-5827

Editor

Institut Français d'Études Andines

\section{Edición impresa}

Fecha de publicación: 1 agosto 2011

Paginación: 411-418

ISSN: 0303-7495

\section{Referencia electrónica}

Chloé Constant, «Relaciones entre internas y guardias en el penal de mujeres de Lima: análisis de las desigualdades de trato », Bulletin de l'Institut français d'études andines [En línea], 40 (2) | 2011

Publicado el 01 febrero 2012, consultado el 07 noviembre 2020. URL : http://journals.openedition.org/ bifea/1550 ; DOI : https://doi.org/10.4000/bifea.1550

\section{(c) (i) (3)}

Les contenus du Bulletin de l'Institut français d'études andines sont mis à disposition selon les termes de la licence Creative Commons Attribution - Pas d'Utilisation Commerciale - Pas de Modification 4.0 International. 


\section{Relaciones entre internas y guardias en el penal de mujeres de Lima: análisis de las desigualdades de trato}

\section{Chloé Constant*}

El proyecto de investigación doctoral del que se trata en el presente artículo está basado en la hipótesis que las estructuras organizacionales de la primera cárcel de mujeres de Lima constituyen un reflejo de la sociedad peruana en términos de reproducción de esquemas de marginación. Se trata de investigar la marginación femenina en cuanto a racismo, clanes, derechos y oportunidades de integración, y asimismo analizar la reproducción de las relaciones de poder, en sus dimensiones física y simbólica, dentro del universo carcelario femenino.

\section{CONTEXTUALIZACIÓN Y METODOLOGÍA}

El trabajo de campo se inició en 2007 y sigue desarrollándose desde febrero 2009 gracias a visitas seguidas al establecimiento penal de mujeres Chorrillos I, más conocido como penal Santa Mónica, del nombre de la patrona de las viudas y las madres solteras (Butler, 2008). En este establecimiento penitenciario se encuentran actualmente aproximadamente 900 presas de derecho común². La elección de este centro penitenciario se basa en la relativa invisibilidad de estas mujeres (Azaola \& Yacamán, 1996) en la sociedad peruana. Los limeños en general conocen esta cárcel ${ }^{3}$ pues fue la primera que el gobierno peruano construyó, a

* Instituto Francés de Estudios Andinos (UMIFRE 17, CNRS-MAEE). E-mail: chloe.constant@gmail. com. Candidata a Doctora por la universidad Paris III- Sorbonne Nouvelle-CREDA.

1 La cifra es aproximada porque el número de internas varía a diario.

2 Se diferencia así a las internas por delito de terrorismo de las internas por otro tipo de delito.

3 Según una encuesta propia. 
mediados del siglo XX, para albergar exclusivamente a delincuentes femeninas. Sin embargo, al revisar los archivos de prensa existentes4, no cabe duda que la imagen que refleja este centro penitenciario a la sociedad peruana en general, y limeña en particular (por su ubicación geográfica), es la de un centro de detención donde se encuentra una mayoría de burriers5. Las autoridades penitenciarias y judiciales suelen difundir la imagen de un centro donde las internas 6 tejen lindas chalinas y efectúan otras tareas típicamente femeninas, en continuidad con los roles de género (Azaola, 1998) socialmente impuestos. En resumidas cuentas, Santa Mónica es una cárcel de mujeres tranquila y casi modelo. Pero ¿traducen estas aserciones una realidad tangible? ¿Qué relaciones de poder rigen este establecimiento? ¿Están las internas tratadas en un modo igualitario por las autoridades penitenciarias? ¿Qué relaciones llevan las internas entre ellas? Fue en base a estas preguntas — entre otros temas abordados - que en enero de 2011 realicé una encuesta con el fin, no solo de reconstruir la trayectoria de vida de las internas para comprender mejor el por qué de su encarcelación, sino también para entender las relaciones de poder que rigen la vida de este centro cerrado, en el cual interactúan internas, personal penitenciario y visitantes externos. Esta encuesta fue llevada en un primer tiempo con un grupo de veinte mujeres que entraban de visita y en un segundo tiempo con treinta y tres internas. El trabajo con las internas, que analizaré en parte aquí, se realizó en un primer tiempo por medio de un cuestionario anónimo, y en un segundo tiempo con una entrevista semidirectiva basada en el cuestionario.

A continuación se presentan las primeras conclusiones del análisis que se realizó sobre las relaciones entre el personal de vigilancia del penal y las internas. Este aspecto de las relaciones de poder será solamente tratado del punto de vista de las internas por el momento, ya que hasta ahora no se ha realizado ninguna encuesta a las guardias del INPE7. Así como lo deja entender el uso del femenino, son exclusivamente mujeres, las «señoritas» como las llaman las internas educadamente, que vigilan el establecimiento. A diario son veinticinco guardias que están a cargo de la vigilancia, pero en realidad raras son las veces que este equipo de trabajo está completo. Según observaciones propias, hay días en los cuales se encuentran solo quince guardias para vigilar a mil internas. Parece entonces indispensable que sea establecido un equilibrio de fuerzas que permite

4 Agradecimientos especiales van dirigidos a la revista Caretas que facilitó desde el año 2007 el acceso al conjunto de sus archivos.

5 El término burrier es un neologismo nacido del encuentro de los términos «burro» en castellano y «courrier» en inglés.

6 Se utilizará aquí el término «internas» para designar a las mujeres privadas de libertad que se encuentran reclusas en el establecimiento penitenciario Santa Mónica, y ello por dos razones. Primero, el término «interno» es el que se encuentra en el Código Penal peruano y que se usa dentro de la administración penitenciaria para designar a las personas privadas de libertad. Segundo, elegí usar este término en mi trabajo por una razón ética, pues ése designa a una persona privada de libertad con un sentido mucho más humano que los términos «reos», «reclusos» o «presos».

7 Instituto Nacional Penitenciario. 
mantener el orden dentro de la prisión. ¿En qué medida se puede afirmar que las relaciones de poder dentro del establecimiento penitenciario Santa Mónica están basadas en un equilibrio que encuentra fundamentos en un modelo social difundido dentro de la sociedad peruana?

\section{ELEMENTOS DE ANÁLISIS DEL TRATO DIFERENCIADO}

Las reacciones de las internas cuando se aborda el tema de las guardias son variadas. Para el presente análisis, se distinguen a tres grupos de internas: las que pretenden no mantener relaciones con las guardias, las que afirman mantener relaciones cordiales con ellas y finalmente las que se quejan abiertamente de ellas. Un primer grupo de mujeres entrevistadas pretende no mantener relaciones con las guardias. Estas internas que se conforman con las reglas impuestas por la dirección del establecimiento penal, son mujeres que han tenido un pasado tranquilo, que no tienen carrera delictiva (Pérez Guadalupe, 2000: 218) y cuya mayoría se declara inocente o víctima de abuso de confianza de parte de terceros. Encarceladas injustamente según varias de ellas, desean evitar cualquier otro problema y no tratan particularmente con las guardias: «Para mí no existen, vivo desconectada, en mi mundo. Ellas pasan cuenta nomás», resume así una joven extranjera. Las internas que conforman este grupo pueden aceptar su condición de reclusa, inclusive muchas de ellas se adaptaron al mundo cerrado y asumen estar privadas de libertad. Sin embargo, por evitar cualquier complicación en su dura vida cotidiana, se someten calladamente al tratamiento penitenciario; se trata de un silencio que esta clase de internas observa no solo hacia las guardias, sino que también marca sus relaciones con la asistenta social y la psicóloga, pero este es otro tema que merecería un análisis que no cabe en el presente artículo. Para este primer grupo de internas, las relaciones con las guardias son definitivamente inexistentes, más allá de la base de relaciones institucionales.

Las relaciones cordiales que caracterizan al segundo grupo de internas están estrechamente vinculadas a lo que denominaría un acuerdo tácito, que une este grupo a las guardias. Este acuerdo sobrepasa la base institucional mencionada arriba (fig. 1). Aquí el factor corrupción resalta por constituir indudablemente el mayor componente de los cimientos de estas relaciones. En efecto, si bien está prohibido el ingreso de numerosos objetos, tales como cuchillos, perfumes o insecticida, estos productos circulan y se venden dentro de la cárcel, y ello gracias a la complicidad de las guardias. Esta operación ilegal de compra-venta se realiza según dos modalidades distintas. A cambio de unos soles, las guardias recogen paquetes que cualquier persona del exterior puede dejar en una tienda ubicada exactamente frente a la prisión. Basta con que una persona externa deje una bolsa con el tendero, con dos a cinco soles, para que la guardia que se compromete a ello la recoja y entre dentro de la cárcel con ella, como si fueran efectos suyos. Paralelamente, las guardias compran productos (ropa, perfumes y otros) a pedido de las internas, e ingresan al penal según la modalidad descrita anteriormente. 


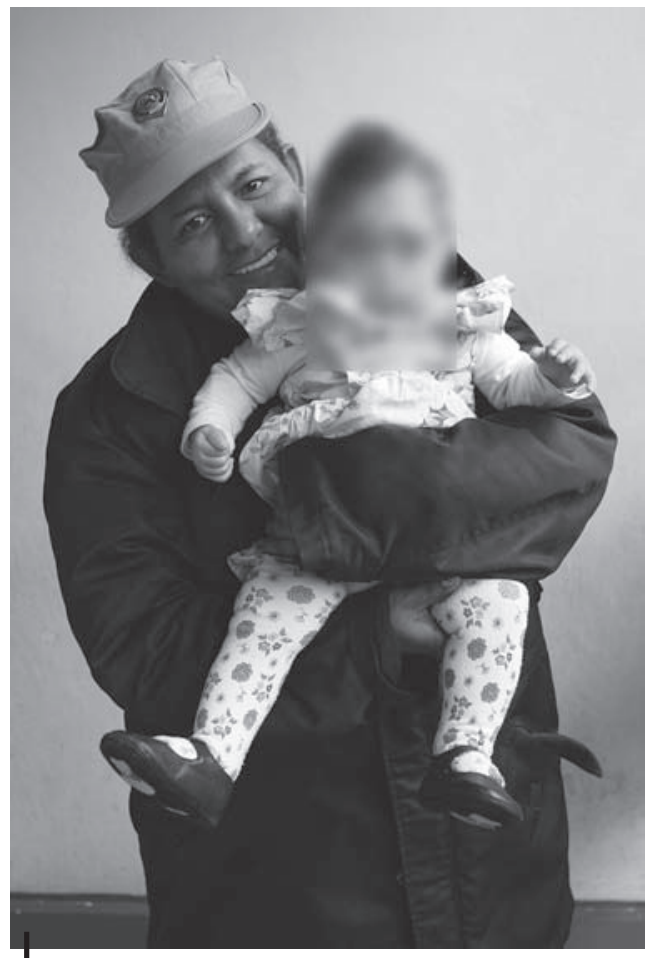

Figura 1 - Las relaciones entre el personal de seguridad y las internas sobrepasan el marco institucional penitenciario. Las internas que mejor se Ilevan con las guardias Ilegan a cierto nivel de confianza; en este caso la madre dejó posar a su hija con una guardia

Fotografía: Santiago Barco, 2007

Notemos que la regularidad de lo que se puede calificar como un verdadero negocio permite a estas funcionarias de sueldo bajo8 mejorar notablemente el nivel de sus ingresos. Las internas que recurren a este tipo de servicio, muy frecuentes según manifiestan, invitan regularmente a las guardias a compartir una gaseosa o unas galletas. Así relata Juana, una joven interna: «Aquí si quieres ser amiga de las INPEs9 tienes que darles. Si no les das, te miran como una rata. Yo le pedía unas sandalias de afuera que acá no pueden entrar. Le das S./ 10 y ella te lo trae, le das S./ 15 y te trae radio. $\mathrm{Y}$ te piden comida. Siempre te piden cosas».

Finalmente las internas como Juana tienen que tratar con las guardias en un juego de equilibrio frágil, ya que el poder siempre lo conserva la autoridad. Por más bajo que se encuentre la figura de la guardia en la escala administrativa penitenciaria, es la representación del poder más cercana a las internas. Por ende, si las internas que solicitan este tipo de «favor» a las guardias quieren seguir disfrutando de estos servicios, bajo ninguna forma pueden derogar a las formas de pago adicionales que solicitan las autoridades mismas.

En la presentación del tercer grupo de mujeres que viene a continuación, veremos en qué medida el racismo, en una aceptación amplia que abarca los sentidos clasista, sexista y nacionalista (Balibar \& Wallerstein, 1988), constituye definitivamente un elemento clave del análisis de las relaciones de poder en el ámbito carcelario. Las mujeres que más se quejan de las guardias corresponden a franjas tradicionalmente marginadas de la población peruana. Por una parte encontramos a las internas más pobres (Durand, 2007: 4558), como esta señora recién encarcelada, que radicaba en el Cercado de Lima:

«No me han dejado entrar faldas ni ropa nueva, ni zapatillas, hay que comprarlas acá. No te dejan entrar baldes ni tina porque dicen que hacemos chicha10 ipero

8 Los guardias del INPE trabajan por turno de 24 horas por un sueldo promedio de S./ 700.

9 El término «INPES» es frecuentemente empleado por las internas para designar a las guardias.

${ }^{10}$ La chicha es una bebida alcohólica que fabrican las internas por maceración de jugo industrial con pan y eventualmente caramelos. 
si acá en la cocina hay! El espejo tampoco te dejan entrar pero en el quiosco venden, lo mismo pasa para el maquillaje. Acá todo te lo venden el doble, una tina si está S./ 5 afuera, acá te lo venden 10».

Esta señora, de origen andino, se encontraba en una situación de pobreza antes de su detención, y tuvo dificultades para tener con ella sus pocas pertenencias, como la tina para lavar su ropa o el balde para realizar los trabajos de limpieza obligatorios. Tiene la posibilidad de comprar estos efectos dentro del penal pero no puede asumir su costo. En su obra reciente, el sociólogo peruano Durand (2007) argumentaba que:

«(...) los pobres, los sectores populares, mantienen su predominio demográfico a pesar de que la pobreza ha cambiado de lugar y de forma, y los patrones de dominación son más indirectos que directos» (2007: 46).

Los trabajos de limpieza son obligatorios dentro de cada pabellón de Santa Mónica. La interna que no desea realizarlos puede pagar a otra que lo hará por ella. El caso de esta señora demuestra, aún más en este tipo de coyuntura, cuán perjudicada está por su estado de pobreza. Como lo argumenta Durand, no por ser provinciana es pobre esta mujer, pues la pobreza alcanzó Lima hace mucho tiempo atrás (Durand, 2007: 45-58), pero por su pobreza las guardias ejercen sobre ella una dominación indirecta. Ya que no le dan la facilidad de ingreso de los productos que necesita, esta señora termina en un dilema por no poder efectuar la tarea obligatoria de limpieza, ni poder pagar a alguien que lo haga por ella. Estamos aquí frente a un caso que ilustra el racismo clasista que existe dentro del penal. En el lado opuesto, las internas que disponen de más facilidades económicas sí acceden a este tipo de favores (consiguen el ingreso de productos) y servicios (pueden contratar a una persona si lo desean).

Paralelamente a los pobres encontramos a otra parte de la población peruana tradicionalmente marginada, que es la población LGTB11. En Santa Mónica muchas son las mujeres lesbianas o bisexuales que se quejan del acoso ejercido por las guardias. El imaginario colectivo peruano no deja de ser influenciado por la Iglesia (La Torré, 2008) y por los medios masivos de comunicación, que difunden una imagen negativa, escandalosa o asquerosa de la población LGTB, como lo subraya el estudio llevado a cabo por el equipo de Carlos Cosme (Cosme et al., 2007). La construcción del prejuicio heteronormativo define un centro y periferias, siendo estas últimas definidas como «'lo que no debe ser'»(Cosme et al., 2007: 95), hecho que los comentarios de la interna Juanita confirman:

«Acá me miraban como si yo fuera no sé qué. Desde que pasé la puerta iacá me miraban con una cara! Hasta los INPEs cuando llegué me miraban raro». Dentro de la prisión, las mujeres «de opción», como suelen llamarlas el personal de tratamiento psicológico, sufren miradas sospechosas a diario. «A mí me enviaron al pabellón C, que no me corresponde12, pero por mi opción lo hicieron», cuenta

11 Lesbiana, Gay, Transexual y Bisexual, según la abreviación comúnmente empleada.

12 El pabellón C alberga supuestamente a las reincidentes, personas mayores o enfermas. El penal es originalmente organizado para acoger a las primerizas, reincidentes, personas mayores o enfermas 
Juanita, de apenas 20 años de edad. Una interna dominicana de 23 años confirma este tipo de práctica:

«Me cambiaron de piso porque decían que tenía novia pero no era. I only had one girlfriend but she left13»〉.

A diario, estas mujeres se sienten especialmente vigiladas por las guardias: «Te miran siempre, siempre están viendo con quién paras y qué haces, son pesadas, esperan que cometas algo que no es para meterte al hueco14», comenta otra joven interna. Finalmente, a través del análisis del grupo de internas conformado por las lesbianas y las bisexuales, las que no cumplen con el modelo heteronormativo impuesto culturalmente, resalta que la heterosexualidad representa una poderosa institución social (Falquet, 2009) que tiene un peso muy importante dentro de la cárcel. Esto nos permite afirmar que opera definitivamente un racismo sexista detrás de las rejas. Por ello las internas homosexuales y bisexuales llevan relaciones muy pesadas con las guardias. En efecto, aparte del encierro, cargan un peso adicional en comparación con las demás internas. A diario tienen que cuidar su conducta, pero no en un sentido de buen comportamiento general, lo que corresponde a cada interna, sino que también tienen que cumplir con el modelo de «centro» (Cosme et al., 2007) impuesto socialmente, pues si su vida sexual es considerada anormal y punible afuera, lo es aún más dentro del mundo cerrado de la cárcel.

El racismo clasista y sexista estructura las relaciones sociales entre peruanos (Bruce, 2007) fuera y dentro de la cárcel. Durante una conversación informal que tuvimos, la directora del Órgano Técnico de Tratamiento15 de Santa Mónica comentó, con tono de lástima y gran lucidez, un aspecto más racial de las relaciones dentro del penal. Declaró que «entre las peruanas mismas hay mucho racismo. Que la blanquita, que la negrita... siendo el Perú un país tan mestizo...». En efecto, varias internas confesaron sentirse discriminadas16, sea por las guardias u otras internas, por su color de piel. Entonces sí existe esta forma de racismo pero afecta a algunas internas de forma individual y no grupal en sus relaciones con las guardias. Para terminar, se observará brevemente el racismo nacionalista. La española María comentó que «no (la)s quieren a las españolas y no se molestan en ocultarlo», lo que confirman varias de sus compatriotas e inclusive peruanas. A partir de estas declaraciones, queda claro que el racismo dentro del penal opera también por nacionalidad, discriminando las internas en una dimensión grupal. Notemos sin embargo que la diferenciación de trato por nacionalidad comprende paralelamente una vertiente beneficiosa, que podremos analizar en otra oportunidad. Finalmente

y madres con hijos en distintos pabellones y pisos. Por el hacinamiento esta organización no está siendo respetada.

13 «Solo tuve a una novia pero salió».

14 El hueco, denominado oficialmente «cuarto de meditación», es una celda apartada donde se castiga a las internas sin dejarlas salir por varios días.

15 El Órgano Técnico de Tratamiento (OTT) es un área del sistema penitenciario que agrupa la educación, la salud, la psicología, la asistencia social, legal y los temas de trabajo.

16 Fue el término exacto en los cuestionarios de la autora. 
el racismo en todas sus matices constituye definitivamente un elemento clave del análisis de las relaciones de poder en el ámbito carcelario.

\section{ALGUNAS CONCLUSIONES}

Se han presentado aquí algunas de las primeras conclusiones del último trabajo de campo realizado. Del análisis de las interacciones y relaciones que existen entre el personal de seguridad y las internas del principal penal de mujeres de Lima, resalta que el espacio carcelario es estrechamente parecido al mundo social exterior en términos organizativos y de poder. La sociedad peruana en su conjunto sigue siendo muy elitista (Durand, 2007) y conservadora (La Torré, 2008), y los mecanismos que rigen la vida cotidiana en los campos institucionales, sociales y políticos, están afectados por una corrupción profundamente arraigada históricamente (Zapata, 2004; Cristóbal, 2004). Estos principios traspasan las puertas del penal para tener efecto dentro de este espacio. Obviamente no se puede generalizar sobre la conducta de las guardias y el poder y las presiones ejercidos por las guardias sobre las internas varían en función de la personalidad misma de los miembros de ambos grupos. Sin embargo los testimonios recogidos durante esta última encuesta revelan cuánto peso tienen los factores sociales, sexuales y raciales en las relaciones de poder del ámbito carcelario.

\section{Referencias citadas}

AZAOLA, E., 1998 - Prisiones para mujeres: un enfoque de género; México: Programa interdisciplinario de Estudios de la Mujer (extraído de internet).

AZAOLA, E. \& YACAMÁN, C. J., 1996 - Las mujeres olvidadas. Un estudio acerca de las cárceles para mujeres en la República Mexicana, 326 pp.; México: El Colegio de México.

BALIBAR, É. \& WALLERSTEIN, I, 1988 - Race Nation Classe. Les identités ambigües, 310 pp.; París: La Découverte.

BRUCE, J., 2007 - Nos habíamos choleado tanto. Psicoanálisis y racismo, 145 pp.; Lima: Universidad de San Martín de Porres.

BUTLER, A., 2008 - Vida de los santos, 192 pp.; Madrid: Libsa.

COSME, C., JAIME, M., MERINO, A. \& ROSALES, J. L., 2007 - La imagen in/decente. Diversidad sexual, prejuicio y discriminación en la prensa escrita peruana, 141 pp.; Lima: IEP.

CRISTOBAL, J., 2004 - Las redes de poder en la cultura de la corrupción. In: Il curso de actualización en Historia y Cultura del Perú (J. M. Cáceres-Olazo Monroy, ed.): 163165; Lima: Universidad Nacional Federico Villareal.

DURAND, F., 2007 - El Perú fracturado. Formalidad, informalidad y economía delictiva, 284 pp.; Lima: Fondo editorial del Congreso del Perú. 
FALQUET, J., 2009 - La règle du jeu. Repenser la co-formation des rapports sociaux de sexe, de classe et de « race » dans la mondialisation néolibérale. In: Sexe, race, classe. Pour une épistémologie de la domination (E. Dorlin, avec la collaboration d'A. Bidet, eds.); París: PUF. Collection Actuel Marx Confrontation.

LA TORRÉ, A., 2008 - Secte, société secrète ou lobby ? L'Opus Dei au Pérou. L'Ordinaire Latino-américain, mai-août, $\mathbf{n}^{\circ}$ 210: 97-119; Toulouse: IPEALT, Université de Toulouse-Le Mirail.

PÉREZ GUADALUPE, J. L., 2000 - La construcción social de la realidad carcelaria, 438 pp.; Lima: PUCP.

ZAPATA, A., 2004 - La corrupción en el Perú de los noventa In: II curso de actualización en Historia y Cultura del Perú (J. M. Cáceres-Olazo Monroy, ed.): 157-161; Lima: Universidad Nacional Federico Villareal. 